1 Does the ratio of $\beta$-1,4-glucosidase $(\mathrm{BG})$ to

$2 \beta-1,4-N$-acetylglucosaminidase $(\mathrm{NAG})$ indicate the relative resource

3 allocation of soil microbes to $\mathrm{C}$ and $\mathrm{N}$ acquisition?

4

5 Taiki Mori ${ }^{1,2^{*}}$, Ryota Aoyagi ${ }^{3}$, Kanehiro Kitayama $^{4}$, and Jiangming $\mathrm{Mo}^{{ }^{*}}$

$6 \quad$ Corresponding authors

$8{ }^{1}$ Key Laboratory of Vegetation Restoration and Management of Degraded Ecosystems,

9 South China Botanical Garden and Guangdong Provincial Key Laboratory of Applied

10 Botany, Chinese Academy of Sciences, Guangzhou, 510650, China

$11{ }^{2}$ Kyushu Research Center, Forestry and Forest Products Research Institute, FFPRI,

12 Kurokami 4-11-16, Kumamoto, 860-0862, Japan

$13{ }^{3}$ Forestry and Forest Products Research Institute, Matsunosato 1, Tsukuba, Ibaraki

$14 \quad 305-8687$, Japan

$15{ }^{4}$ Graduate School of Agriculture, Kyoto University, Kitashirakawa Oiwake-cho

16 606-8502, Sakyo-ku, Kyoto, Japan

18 Corresponding authors

19 Taiki Mori: taikimori7@gmail.com

20 Jiangming Mo: mojm@scib.ac.cn

\title{
22 Abstract
}

23 The ratio of $\beta-1,4$-glucosidase (BG) to $\beta-1,4-\mathrm{N}$-acetylglucosaminidase (NAG) activity

24 (BG:NAG ratio) is often used as an indicator of the relative resource allocation of soil 
25 microbes to $\mathrm{C}$ acquisition compared with $\mathrm{N}$. An increasing number of recent studies

26 have used this index to assess the nutrient status of microbes. However, the validity of

27 this index for assessing the nutrient status of microbes is not well tested. In this study,

28 we collected published data and tested that validity by investigating whether $\mathrm{N}$

29 fertilization elevated the BG:NAG ratio, assuming that microbes reduce their allocation

30 to the $\mathrm{N}$-acquiring enzyme (NAG) under $\mathrm{N}$-enriched conditions. Of the data points,

$3154 \%(82 / 151)$ did not support the hypothesis because those studies showed lower

32 BG:NAG ratios in N-enriched soils than under ambient conditions, especially when the

33 ambient BG:NAG ratio was higher than $2.0(77 \%, 59 / 77)$. This suggests that the

34 BG:NAG ratio does not always indicate the microbial status for $\mathrm{C}$ or $\mathrm{N}$ limitation.

35 Rather, we hypothesized that the decomposition stage explained the variation in

36 BG:NAG because $\mathrm{N}$ addition accelerates decomposition, and the BG:NAG ratio is

37 lower at later stages of decomposition due to the dominance of NAG-targeting C (chitin

38 or peptidoglycan). A negative correlation of BG:NAG ratio with polyphenol oxidase

39 activity, which increases with decomposition, supported our hypothesis.

$41 \quad$ Key words: $\quad \beta-1,4$-glucosidase $\quad(B G) ; \quad \beta-1,4-N$-acetylglucosaminidase $\quad$ (NAG);

42 decomposition; enzymatic stoichiometry; meta-analysis; nitrogen fertilization

\section{Introduction}

45 Prior authors have suggested that the relative resource allocation of soil microbes to

46 acquire energy and nutrients can be expressed as the ratio of extracellular enzyme

47 activity targeting carbon $(\mathrm{C})$, nitrogen $(\mathrm{N})$, and phosphorus (P) (Sinsabaugh et al., 2008,

48 2009; Waring et al., 2014). The ratio of $\beta$-1,4-glucosidase (BG) to 
$49 \beta-1,4-\mathrm{N}$-acetylglucosaminidase (NAG) (BG:NAG ratio) is often used as an indicator of

50 the relative resource allocation of microbes to $\mathrm{C}$ acquisition compared with $\mathrm{N}$

51 acquisition (Turner \& Wright, 2014; Waring et al., 2014; Zhou et al., 2017; Chen et al.,

52 2018). Recently, increasing numbers of studies have used the BG:NAG ratio to assess

53 nutrient limitation or the status of microbes, assuming that a lower BG:NAG ratio

54 indicates $\mathrm{N}$ shortage/ $\mathrm{N}$ limitation (hereafter, the ecoenzymatic stoichiometry hypothesis;

55 (Sinsabaugh et al., 2008; Waring et al., 2014; Moorhead et al., 2016; Chen et al., 2018;

56 Mori et al., 2018a; Wang et al., 2018)). Nevertheless, this hypothesis has not been tested

57 sufficiently, and several studies examining it have reported inconsistent results (Mori et

58 al., 2018a; Rosinger et al., 2019; Mori, 2020). The validity of the BG:NAG ratio as a

59 measure of the nutrient status of microbes needs to be tested by synthesizing

60 accumulated data.

61 The ecoenzymatic stoichiometry hypothesis can be tested in $\mathrm{N}$ fertilization

62 experiments. If the BG:NAG ratio really indicates the nutrient status of microbes, $\mathrm{N}$

63 fertilization will elevate it because microbes can get $\mathrm{N}$ directly from the fertilizer and

64 reduce the allocation to $\mathrm{N}$-acquiring enzymes, i.e., NAG. Furthermore, the response of

65 the BG:NAG ratio to $\mathrm{N}$ fertilization would be smaller under $\mathrm{N}$-rich conditions because

66 the decrease in NAG activity would be smaller. Accordingly, the predicted distribution

67 of data could be drawn as shown in Fig. 1. In the present study, we collected published

68 papers reporting the impact of $\mathrm{N}$ fertilization on the activity of BG and NAG and

69 compared the BG:NAG ratio in surface soils before and after $\mathrm{N}$ fertilization.

70 We also established an alternative hypothesis, which stems from a completely

71 different mechanism: the BG:NAG ratio does not indicate the relative allocation of

72 microbes to $\mathrm{C}$ and $\mathrm{N}$, but the source of the $\mathrm{C}$ resources (substrate) for microbes (Mori, 
73 2020). This hypothesis is plausible for the following two reasons: (i) enzyme activity

74 varies depending on the relative substrate availability [several studies have reported that

75 the addition of a substrate caused elevation of enzyme activity that targets the added

76 substrate (Shackle et al., 2000)], and (ii) both BG and NAG can be produced for

77 acquiring C (Mori et al., 2018a; Wang et al., 2018; Mori, 2020). When cellulose (i.e., a

78 BG-targeting $\mathrm{C}$ compound) is the dominant $\mathrm{C}$ resource in soil, microbes utilize more

79 cellulose than chitin and peptidoglycan (a NAG-targeting C compound), leading to

80 higher BG activity and a higher BG:NAG ratio (Mori, 2020). By contrast, chitin and

81 peptidoglycan-dominant conditions cause microbes to utilize more chitin and

82 peptidoglycan than cellulose, resulting in higher NAG activity and a lower BG:NAG

83 ratio (Mori, 2020). As decomposition progresses, the relative abundance of cellulose

84 decreases, whereas chitin and peptidoglycan becomes more abundant because chitin and

85 peptidoglycan derived from microbial death is supplied to the soil via microbial

86 turnover (see Fig. 2). As a result, NAG becomes a more dominant C-acquiring enzyme

87 than BG. Therefore, our alternative hypothesis predicts that the BG:NAG ratio is lower

88 when abundant soil organic matter is progressively decomposed (Fig. 2). According to

89 this hypothesis, $\mathrm{N}$ fertilization can reduce the BG:NAG ratio, in contrast to the

90 enzymatic stoichiometry hypothesis, because $\mathrm{N}$ enrichment is expected to stimulate

91 organic matter decomposition under N-poor conditions (Hobbie, 2005). Furthermore,

92 the hypothesis predicts that the BG:NAG ratio will be negatively correlated with the

93 activity of polyphenol oxidase (PPO), a well-measured ecoenzyme that oxidizes lignin

94 or humus and increases as decomposition progresses (Moorhead \& Sinsabaugh, 2006;

95 Sinsabaugh \& Shah, 2011). To validate the prediction, data on PPO activity were also 
96 collected from the literature. As $\mathrm{N}$ fertilization often has negative effects on PPO, we

97 analyzed the correlations using only N-unfertilized data.

99 Material and Methods

100 Jian et al. (2016) published a meta-analysis reporting the impact of $\mathrm{N}$ fertilization on

101 ecoenzymes, which comprehensively collected data on the response of ecoenzyme

102 activity, including $\mathrm{BG}$ and NAG, to $\mathrm{N}$ fertilization through 2015 , so we used the

103 reported data in our analysis. Then, we searched the Web of Science for papers

104 published later than 2015 using the following combinations of key words: (NAG OR

105 chitinase OR $\beta$-1,4-N-acetyl- $\beta$-glucosaminidase OR " $\mathrm{N}$-acetyl $\beta$-glucosaminidase" OR

106 glucosaminidase) $A N D$ (BG OR $\beta$ G OR $\beta$-1,4-glucosidase OR glucosidase) $A N D$

107 ("nitrogen add*” OR "N add*” OR "nitrogen elevat*” OR "N elevat*” OR "nitrogen

108 fertiliz*" OR "N fertiliz*” OR “nitrogen appl*” OR "N appl*”OR "nitrogen enrich*”

109 OR "N enrich*") (time span 2015-2018). We collected 151 data points from 40 papers.

110 The relationship between the BG:NAG ratio under ambient conditions and that under

$111 \mathrm{~N}$-enriched (fertilized) conditions was examined. If the paper reported PPO activity, we

112 also recorded that. Pearson's test was used to test the correlations between enzyme

113 activities and the BG:NAG ratio. All statistical analyses were performed using $\mathrm{R}$ ver.

$114 \quad 3.4 .1$ or 3.4.4 (R Core Team, 2018).

\section{Results and Discussion}

117 A large proportion of the synthesized data did not support the ecoenzymatic

118 stoichiometry hypothesis, although the distribution of the data was close to the predicted

119 pattern (Fig. 1) when the ambient BG:NAG ratio was low (Fig. 3b). Of 151 data points, 
$12082(54 \%)$ indicated lower BG:NAG ratios in N-enriched soils than under ambient

121 conditions (i.e., the data points are below the solid line in Fig. 3), especially when the

122 ambient BG:NAG ratio was higher than $2.0(77 \%, 59 / 77)$. According to the chi-square

123 test, a higher positive response ratio occurred when the ambient BG:NAG ratio was

$124<2.0(P<0.001)$. The ecoenzymatic stoichiometry hypothesis cannot explain the

125 lowered BG:NAG ratio in N-fertilized soils because, according to that hypothesis, the

126 result indicates that $\mathrm{N}$ fertilization enhances the $\mathrm{N}$ shortage, which is contradictory. Our

127 meta-analysis suggested that the BG:NAG ratio does not always indicate the nutrient

128 status of soil microbes.

129 Other studies have also reported results inconsistent with the ecoenzymatic 130 stoichiometry hypothesis (also see a perspective by Mori, 2020). Waring et al. (2014)

131 collected 17 studies of N-rich tropical ecosystems and found that the mean BG:NAG

132 ratio in these sites was not significantly different from the global average. In a lowland

133 tropical rainforest in Bornean Malaysia, Mori et al. (2018a) reported that the BG:NAG

134 ratio was similar to (or even slightly smaller than) the global average, although the

135 forest is considered $\mathrm{N}$ rich (Aoyagi \& Kitayama, 2016). This could be explained by the

136 following hypothetical mechanism: under N-rich conditions, microbes produce

137 NAG-targeting $\mathrm{C}$ because chitin and peptidoglycan, whose terminal reaction is

138 catalyzed by NAG (Waring et al., 2014), contains both C and N (Mori et al., 2018a,

139 2018b; Wang et al., 2018). Several papers have supported our idea, reporting high NAG

140 activity with a lack of a response of the NAG activity or BG:NAG ratio to N

141 fertilization (see the meta-analysis by Jian et al. 2016).

142 Our alternative hypothesis was supported by the collected data. As predicted by

143 the hypothesis, PPO activity was negatively correlated with the ambient BG:NAG ratio 
144 in our dataset (Fig. 4). The result supports the idea that the BG:NAG ratio shows the 145 decomposition stage. That is, as decomposition progresses, the BG:NAG ratio decreases

146 because the abundance of chitin and peptidoglycan becomes dominant relative to that of

147 cellulose (Fig. 2). Our alternative hypothesis can explain how $\mathrm{N}$ fertilization decreases

148 the BG:NAG ratio and why lower BG:NAG ratios were mainly observed when the

149 ambient BG:NAG ratios were $>2.0$ (Fig. 3). At a relatively early stage of the

150 decomposition process, where the cellulose content is relatively large and dominates the

151 microbial $\mathrm{C}$ resources (higher $\mathrm{BG}: \mathrm{NAG}$ ratio), $\mathrm{N}$ fertilization can accelerate

152 decomposition and microbial activity (Yoshitake et al., 2007). In such a case, the added

$153 \mathrm{~N}$ can change the $\mathrm{C}$ source of microbes to more microbial dead body-derived $\mathrm{C}$ (chitin

154 and peptidoglycan dominant, NAG-targeting C), resulting in a lower BG:NAG ratio

155 under $\mathrm{N}$-added conditions. Conversely, at a later stage of decomposition, $\mathrm{N}$ addition

156 generally suppresses organic matter decomposition (Fog, 1988; Knorr et al., 2005;

157 Janssens et al., 2010), and a lower BG:NAG ratio caused by $\mathrm{N}$ fertilization should be 158 observed less often (Fig. 3).

159 Although some of our data did not support the ecoenzymatic stoichiometry 160 hypothesis, we cannot completely reject the hypothesis. When the ambient BG:NAG

161 ratios are $<2.0$, the distribution of the data was close to the pattern predicted by that

162 hypothesis (Fig. 1), and 69\% (51/74) of the data points showed higher BG:NAG ratios

163 in $\mathrm{N}$-fertilized soils than under ambient conditions (Fig. 3). It is possible that the

164 ecoenzymatic stoichiometry hypothesis is true when the NAG activity is relatively high,

165 whereas another mechanism (such as our alternative hypothesis) controls the BG:NAG

166 ratio in ecosystems with high BG:NAG ratios. The meta-analysis approach is unable to

167 test this idea. We need other approaches, such as a laboratory experiment monitoring 
168 the BG:NAG ratio of organic matter over the course of decomposition under both

169 manipulated N-shortage and N-rich conditions.

170 In summary, we demonstrated that the BG:NAG ratio may not always indicate

171 the nutrient status of microbes, as previously suggested (Rosinger et al., 2019; Mori,

172 2020), at least when the initial BG:NAG ratio exceeds 2.0. Our dataset also indicated

173 that the stage of decomposition can explain variation in BG:NAG.

\section{Acknowledgement}

176 This study was financially supported by a grant from environmental research aid, The

177 Sumitomo Foundation 153082 and Grant-in-Aid for JSPS Postdoctoral Fellowships for

178 Research Abroad (28:601) to T Mori.

179

180 Statement of authorship: TM conceived this study. TM and RA wrote the first draft of

181 the manuscript. All of the authors contributed to the discussion and writing of the 182 manuscript.

\section{Conflict of interest}

185 We declare that we do not have any conflicts of interest.

\section{References}

188 Aoyagi R. \& Kitayama K. (2016). Nutrient allocation among plant organs across 13 tree 189 species in three Bornean rain forests with contrasting nutrient availabilities.

$190 \quad$ Journal of Plant Research, 129(4), 1-10.

191 https://doi.org/10.1007/s10265-016-0826-z. 
192 Chen H., Li D., Zhao J., Zhang W., Xiao K. \& Wang K. (2018). Nitrogen addition

193 aggravates microbial carbon limitation: Evidence from ecoenzymatic

194 stoichiometry. Geoderma, 329(January), 61-64.

195 https://doi.org/10.1016/j.geoderma.2018.05.019.

196 Fog K. (1988). The effect of added nitrogen on the rate of decomposition of organic

197 matter. Biological Reviews, 63(3), 433-462.

198 https://doi.org/10.1111/j.1469-185X.1988.tb00725.x.

199 Hobbie S. E. (2005). Contrasting effects of substrate and fertilizer nitrogen on the early

$200 \quad$ stages of litter decomposition. Ecosystems, 8(6), 644-656.

$201 \quad$ https://doi.org/10.1007/s10021-003-0110-7.

202 Janssens I., Dieleman W., Luyssaert S., Subke J., Reichstein M., Ceulemans R., ... Law

203 B. (2010). Reduction of forest soil respiration in response to nitrogen deposition.

$204 \quad$ Nature Geoscience, 3(5), 315-322. https://doi.org/10.1038/ngeo844.

205 Jian S., Li J., Chen J., Wang G., Mayes M. A., Dzantor K. E., ... Luo Y. (2016). Soil

206 extracellular enzyme activities, soil carbon and nitrogen storage under nitrogen

207 fertilization: A meta-analysis. Soil Biology and Biochemistry, 101, 32-43.

208 https://doi.org/10.1016/j.soilbio.2016.07.003.

209 Knorr M., Frey S. \& Curtis P. (2005). Nitrogen additions and litter decomposition: A

210 meta analysys. Ecology, 86(12), 3252-3257. https://doi.org/10.1890/05-0150.

211 Moorhead D. L. \& Sinsabaugh R. L. (2006). A theoretical model of litter decay and

212 microbial interaction. Ecological Monographs, 76(2), 151-174.

213 Moorhead D. L., Sinsabaugh R. L., Hill B. H. \& Weintraub M. N. (2016). Vector

214 analysis of ecoenzyme activities reveal constraints on coupled C, N and P 
215 dynamics. Soil Biology and Biochemistry, 93, 1-7.

216 https://doi.org/10.1016/j.soilbio.2015.10.019.

217 Mori T. (2020). Does ecoenzymatic stoichiometry really determine microbial nutrient

218 limitations? Soil Biology and Biochemistry, 146(April), 107816.

219 https://doi.org/10.1016/j.soilbio.2020.107816.

220 Mori T., Imai N., Yokoyama D. \& Kitayama K. (2018a). Soil Science and Plant

221 Nutrition Effects of nitrogen and phosphorus fertilization on the ratio of activities

222 of carbon-acquiring to nitrogen-acquiring enzymes in a primary lowland tropical

223 rainforest in Borneo, Malaysia. Soil Science and Plant Nutrition, 00(00), 1-4.

224 https://doi.org/10.1080/00380768.2018.1498286.

225 Mori T., Lu X., Aoyagi R. \& Mo J. (2018b). Reconsidering the phosphorus limitation of

226 soil microbial activity in tropical forests. Functional Ecology, 32(December 2017),

227 1145-1154. https://doi.org/10.1111/1365-2435.13043.

228 R Core Team (2018). R: A language and environment for statistical computing. R

229 Foundation for Statistical Computing, Vienna, Austria. URL

$230 \quad$ https://www.R-project.org/. 2018.

231 Rosinger C., Rousk J. \& Sandén H. (2019). Can enzymatic stoichiometry be used to

232 determine growth-limiting nutrients for microorganisms? - A critical assessment in

233 two subtropical soils. Soil Biology and Biochemistry.

234 https://doi.org/10.1016/j.soilbio.2018.10.011.

235 Shackle V. J., Freeman C. \& Reynolds B. (2000). Carbon supply and the regulation of

236 enzyme activity in constructed wetlands. Soil Biology and Biochemistry, 32,

$237 \quad 1935-1940$. 
238 Sinsabaugh R. L., Hill B. H. \& Follstad Shah J. J. (2009). Ecoenzymatic stoichiometry

239 of microbial organic nutrient acquisition in soil and sediment. Nature, 462(7274),

240 795-8. https://doi.org/10.1038/nature08632.

241 Sinsabaugh R. L. \& Shah J. J. F. (2011). Ecoenzymatic stoichiometry of recalcitrant

242 organic matter decomposition: The growth rate hypothesis in reverse.

243 Biogeochemistry, 102(1), 31-43. https://doi.org/10.1007/s10533-010-9482-x.

244 Sinsabaugh R. L., Lauber C. L., Weintraub M. N., Ahmed B., Allison S. D., Crenshaw

245 C., ... Zeglin L. H. (2008). Stoichiometry of soil enzyme activity at global scale.

246 Ecology Letters, 11(11), 1252-1264.

247 https://doi.org/10.1111/j.1461-0248.2008.01245.x.

248 Turner B. L. \& Wright S. J. (2014). The response of microbial biomass and hydrolytic

249 enzymes to a decade of nitrogen, phosphorus, and potassium addition in a lowland

$250 \quad$ tropical rain forest. Biogeochemistry, 117(1), 115-130.

251 https://doi.org/10.1007/s10533-013-9848-y.

252 Wang C., Lu X., Mori T., Mao Q., Zhou K., Zhou G., ... Mo J. (2018). Responses of

253 soil microbial community to continuous experimental nitrogen additions for 13

254 years in a nitrogen-rich tropical forest. Soil Biology and Biochemistry, 121.

255 https://doi.org/10.1016/j.soilbio.2018.03.009.

256 Waring B. G., Weintraub S. R. \& Sinsabaugh R. L. (2014). Ecoenzymatic stoichiometry

257 of microbial nutrient acquisition in tropical soils. Biogeochemistry, 117(1),

258 101-113. https://doi.org/10.1007/s10533-013-9849-x.

259 Yoshitake S., Uchida M., Koizumi H. \& Nakatsubo T. (2007). Carbon and nitrogen

260 limitation of soil microbial respiration in a High Arctic successional glacier 
261 foreland near Ny-??lesund, Svalbard. Polar Research, 26(1), 22-30.

262 https://doi.org/10.1111/j.1751-8369.2007.00001.x.

263 Zhou Z., Wang C. \& Jin Y. (2017). Stoichiometric responses of soil microflora to

264 nutrient additions for two temperate forest soils. Biology and Fertility of Soils, 53,

265 397-406. https://doi.org/10.1007/s00374-017-1188-y.

266

267 


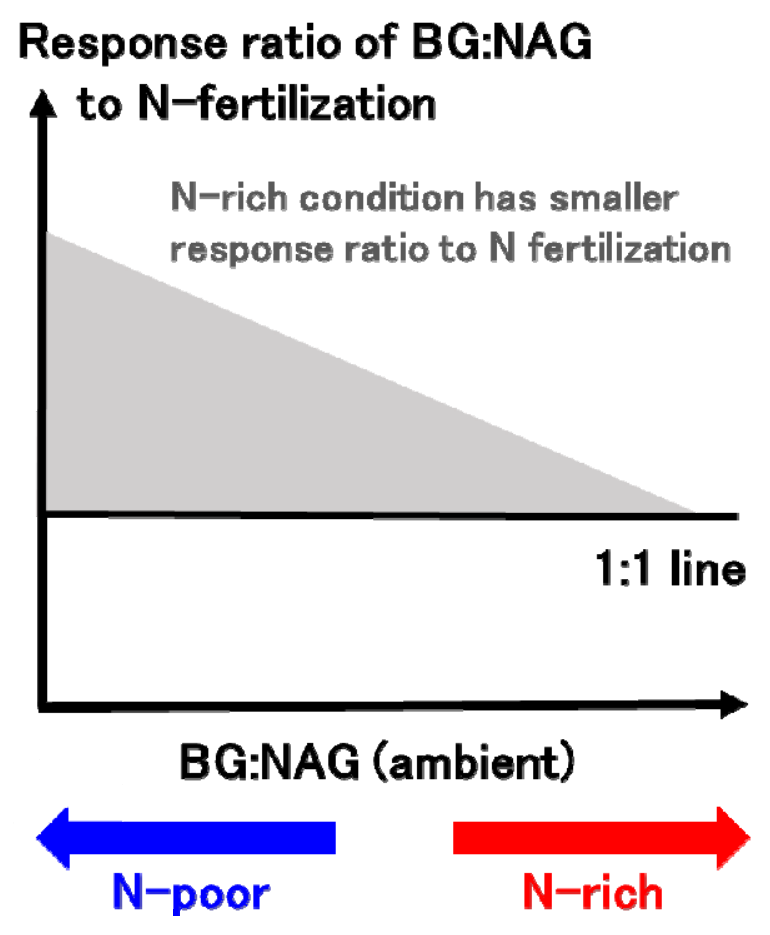

Fig. 1. The predicted relationship between the BG:NAG ratio under ambient conditions and the response ratio of the $\mathrm{BG}: \mathrm{NAG}$ ratio to $\mathrm{N}$ fertilization according to the enzymatic stoichiometry hypothesis. Data points would be plotted above the 1:1 line because $\mathrm{N}$ fertilization does not lower the BG:NAG ratio. N-rich conditions (i.e., a low BG:NAG ratio) showed a lower response ratio to $\mathrm{N}$ fertilization. 


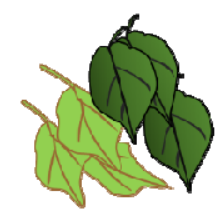

High cellulose

\section{The ratio of \\ plant-derived C}

The ratio of

microbial dead body-derived C

Progressed

Decomposition stage

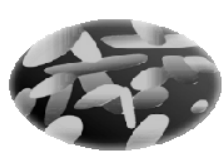

High chitin and peplidoglycan

Initial

BG: NAG

Microbes utilize more cellulose as $\mathbf{C}$ resources.

Fig. 2. Our new hypothesis explaining what the BG:NAG ratio shows. As microbial activity accelerates, the ratio of plant-derived $\mathrm{C}$ in the total $\mathrm{C}$ pool decreases, while the ratio of microbial dead body-derived $\mathrm{C}$ in the total $\mathrm{C}$ pool increases. As a result, NAG becomes the more dominant $\mathrm{C}$-acquiring enzyme compared with $\mathrm{BG}$, which leads to a decrease in the BG:NAG ratio. 


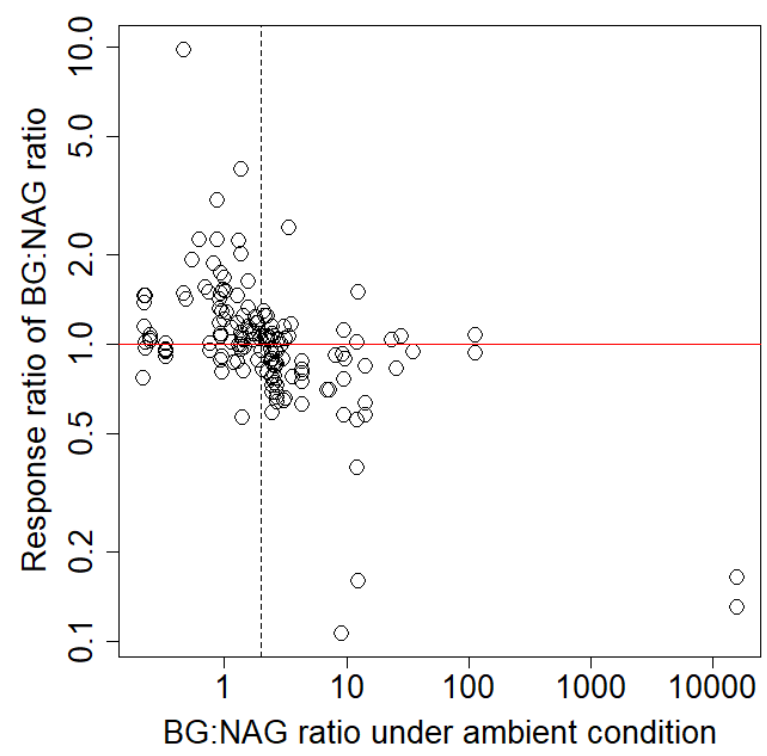

Fig. 3. The correlation between the BG:NAG ratio under ambient conditions with the response of the BG:NAG ratio to $\mathrm{N}$ fertilization. The solid red line is the 1:1 line. The dashed line represents the ambient condition under which the BG:NAG is 2.0. Of 151 data points, $82(54 \%)$ showed lower BG:NAG ratios under ambient conditions. When the ambient BG:NAG ratio was higher than 2.0, 59 of $77(77 \%)$ data points showed a lower BG:NAG ratio under ambient conditions. 


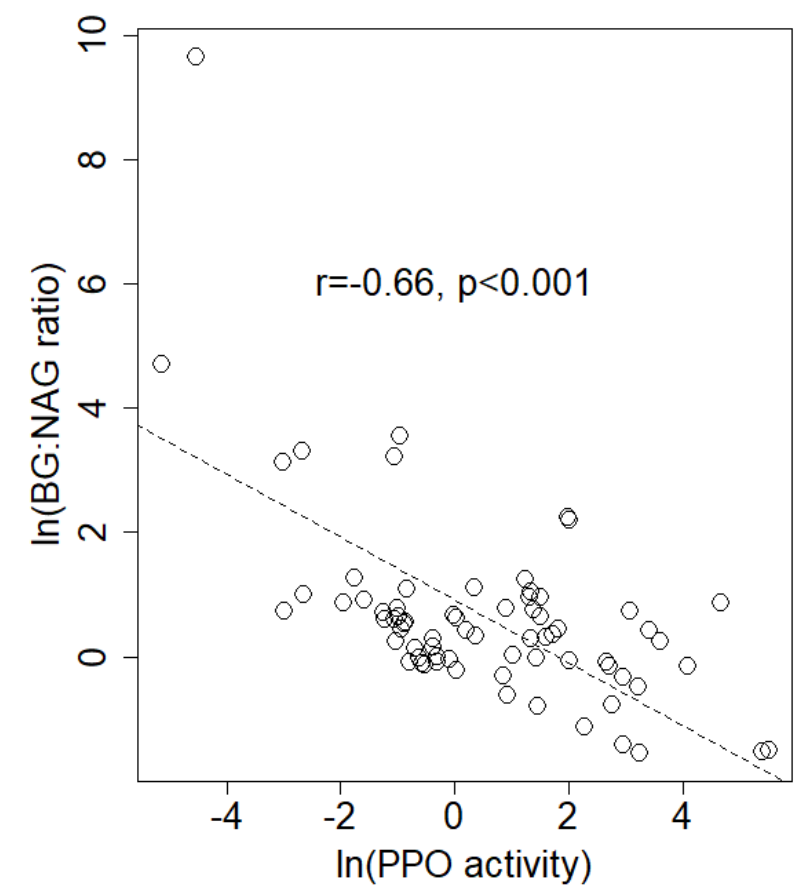

Fig. 4. The correlation of $\ln (\mathrm{PPO}$ activity under ambient conditions) with $\ln (\mathrm{BG}: \mathrm{NAG}$ ratio under ambient conditions). 